Check for updates

Cite this: J. Mater. Chem. A, 2021, 9, 2471

DOI: $10.1039 / \mathrm{d} 1 \mathrm{ta} 90014 \mathrm{e}$

rsc.li/materials-a

\section{Correction: Ruthenium oxide coated ordered mesoporous carbon nanofiber arrays: a highly bifunctional oxygen electrocatalyst for rechargeable $\mathrm{Zn}$-air batteries}

\author{
Ziyang Guo, ${ }^{\text {a }}$ Chao Li, ${ }^{a}$ Wangyu Li, ${ }^{a}$ Hua Guo, ${ }^{a}$ Xiuli Su, ${ }^{a}$ Ping He, ${ }^{b}$ Yonggang Wang ${ }^{\star a}$ \\ and Yongyao $\mathrm{Xia}^{\mathrm{a}}$
}

Correction for 'Ruthenium oxide coated ordered mesoporous carbon nanofiber arrays: a highly bifunctional oxygen electrocatalyst for rechargeable Zn-air batteries' by Ziyang Guo et al., J. Mater. Chem. A, 2016, 4, 6282-6289, DOI: 10.1039/C6TA02030E.

The authors regret a minor error in Fig. 5 of the published article, where it is found that Fig. $5 \mathrm{c}$ is a repetition of Fig. $5 \mathrm{~d}$. The error was attributable to the fact that the authors inadvertently copied Fig. $5 \mathrm{~d}$ and pasted it as Fig. $5 \mathrm{c}$ during the figure preparation process. The corrected Fig. $5 \mathrm{c}$ should appear as shown below. Ziyang Guo detected this error when he re-read this paper recently. This minor error has no effect on the conclusions of the paper. Furthermore, the authors state that the raw data of Fig. $5 \mathrm{c}$ are available from the first author (Z. G.) and/or the corresponding author (Y. W.) upon request. The authors apologize for any inconvenience caused by this error.

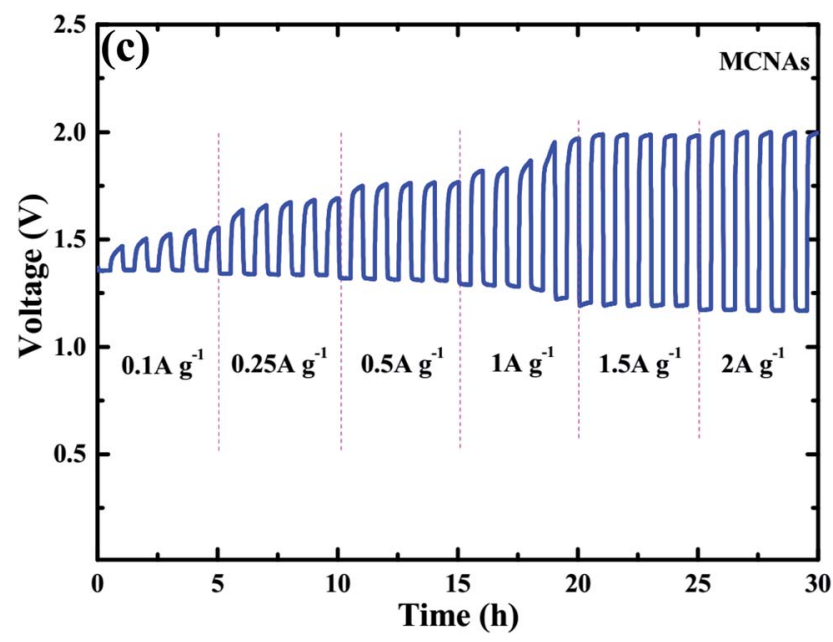

Fig. 5 (c) The discharge/charge curves of Zn-air batteries using bare MCNAs catalyst at different current densities.

The Royal Society of Chemistry apologises for these errors and any consequent inconvenience to authors and readers.

\footnotetext{
${ }^{a}$ Department of Chemistry, Shanghai Key Laboratory of Molecular Catalysis and Innovative Materials, Institute of New Energy, iChEM (Collaborative Innovation Center of Chemistry for Energy Materials), Fudan University, Shanghai 200433, China. E-mail: ygwang@fudan.edu.cn; Fax: +86-21-51630318; Tel: +86-21-51630318

${ }^{b}$ Center of Energy Storage Materials \& Technology, College of Engineering and Applied Sciences, National Laboratory of Solid State Microstructures and Collaborative Innovation Center of Advanced Microstructures, Nanjing University, Nanjing 210093, China
} 